\title{
ITPR3 gene haplotype is associated with cervical squamous cell carcinoma risk in Taiwanese women
}

\author{
Yuh-Cheng Yang ${ }^{1,4, *}$, Tzu-Yang Chang ${ }^{3, *}$, Tze-Chien Chen ${ }^{1}$, Wen-Shan Lin ${ }^{3}$, Shih- \\ Chuan Chang ${ }^{3}$, Yann-Jinn Lee ${ }^{2,3,5,6,7}$ \\ ${ }^{1}$ Department of Gynecology and Obstetrics, MacKay Memorial Hospital, Taipei City \\ ${ }^{2}$ Department of Pediatric Endocrinology, MacKay Children's Hospital, Taipei City \\ ${ }^{3}$ Department of Medical Research, MacKay Memorial Hospital, New Taipei City \\ ${ }^{4}$ Departments of Gynecology and Obstetrics, Pediatrics, School of Medicine, College of Medicine, Taipei Medical University, \\ Taipei City \\ ${ }^{5}$ Departments of Gynecology and Obstetrics, Pediatrics, School of Medicine, College of Medicine, Taipei Medical University, \\ Taipei City \\ ${ }^{6}$ Institute of Biomedical Sciences Mackay Medical College, New Taipei City, Taiwan \\ ${ }^{7}$ Department of Medicine, Mackay Medical College, New Taipei City, Taiwan \\ *These authors contributed equally to this work. \\ Correspondence to: Yann-Jinn Lee, email: yannlee@mmh.org.tw, kylechang@mmh.org.tw
}

Keywords: cervical cancer, HPV, immunity, ITPR3, polymorphism

Received: June 15, $2016 \quad$ Accepted: December 15, 2016

Published: December 28, 2016

\section{ABSTRACT}

Host immunogenetic background plays an important role in human papillomavirus (HPV) infection and cervical cancer development. Inositol 1,4,5-triphosphate receptor type 3 (ITPR3) is essential for both immune activation and cancer pathogenesis. We aim to investigate if ITPR3 genetic polymorphisms are associated with the risk of cervical cancer in Taiwanese women. ITPR3 rs3748079 A/G and rs2229634 C/T polymorphisms were genotyped in a hospital-based study of 462 women with cervical squamous cell carcinoma (CSCC) and 921 age-matched healthy control women. The presence and genotypes of HPV in CSCC was determined. No significant association of individual ITPR3 variants were found among controls, CSCC, and HPV-16 positive CSCC. However, we found a significant association of haplotype AT between CSCC and controls (OR $=2.28,95 \%$ CI 1.31-3.97, $P=2.83 \times 10^{-3}$ ) and the OR increased further in CSCC patients infected with HPV-16 (OR $=2.89,95 \%$ CI 1.55-5.37, $P=4.54 \times$ $10^{-4}$ ). The linkage disequilibrium analysis demonstrated that ITPR3 association with CSCC was independent of HLA-DRB1 alleles. In conclusion, these findings suggest that AT haplotype in the ITPR3 gene may serve as a potential marker for genetic susceptibility to CSCC.

\section{INTRODUCTION}

Cervical cancer is the fourth most common cancer in women worldwide. In Taiwan, cervical cancer also poses a major public health concern, with nearly 2700 women were diagnosed with cervical cancer each year [1]. Infection with oncogenic human papillomavirus (HPV) is necessary for the development of cervical cancer [2]. However, the majority of women infected with HPV do not progress into cervical cancer, suggesting that other factors are required for the cancer to develop. A potential cofactor is the individual immune system, which may play a pivotal role in viral clearance and involve in the cervical cancer development.

The inositol 1,4,5-triphosphate receptor type 3 (ITPR3), an intracellular $\mathrm{Ca}^{2+}$ release channel on the endoplasmic reticulum membrane, is responsive to the binding of a second messenger inositol 1,4,5-triphosphate (IP3) [3]. IP3 is phosphorylated to become IP4 by inositol 1,4,5-triphosphate 3-kinase C (ITPKC) and negatively regulates T-cell receptor signal transduction [4]. A study reported by our group revealed that a genetic variant 
Table 1: Genotype and allele frequencies of the ITPR3 $r$ s3748079 $A / G$ polymorphism in controls and in women with CSCC and those with HPV-16 positive CSCC*

\begin{tabular}{cccccccc}
\hline & $\begin{array}{c}\text { Controls } \\
(\mathbf{N}=\mathbf{9 2 1})\end{array}$ & $\begin{array}{c}\text { CSCC } \\
(\mathbf{N}=\mathbf{4 6 2})\end{array}$ & $\begin{array}{c}\text { HPV-16 } \\
\text { positive } \\
\text { CSCC } \\
(\mathbf{N}=\mathbf{2 4 5})\end{array}$ & CSCC & HPV-16 positive CSCC \\
\hline & No. (\%) & No. (\%) & No. $(\%)$ & P value $\left(\chi^{2}\right)$ & OR (95\% CI) & P value $\left(\chi^{2}\right)$ & OR (95\% CI) \\
\hline Genotype & & & & $0.34(2.16)$ & & $0.77(0.53)$ & \\
A/A & $40(4.3)$ & $28(6.1)$ & $13(5.3)$ & & $1.42(0.86-2.33)$ & & $1.23(0.65-2.35)$ \\
A/G & $301(32.7)$ & $154(33.3)$ & $82(33.5)$ & & $1.03(0.81-1.31)$ & $1.04(0.77-1.40)$ \\
G/G & $580(63.0)$ & $280(60.6)$ & $150(61.2)$ & & $0.90(0.72-1.14)$ & & $0.93(0.69-1.24)$ \\
Allele & & & & $0.22(1.53)$ & & $0.51(0.43)$ & \\
A & $381(20.7)$ & $210(22.7)$ & $108(22.0)$ & & $1.13(0.93-1.36)$ & & $1.08(0.85-1.38)$ \\
G & $1461(79.3)$ & $714(77.3)$ & $382(78.0)$ & & $0.89(0.73-1.07)$ & & $0.92(0.72-1.17)$ \\
\hline
\end{tabular}

* ITPR3 = inositol 1,4,5-trisphosphate receptor type 3; CSCC = cervical squamous cell carcinoma; HPV = human papillomavirus; $\mathrm{OR}=$ odds ratio; $\mathrm{CI}=$ confidence interval.

rs 28493229 of the ITPKC gene is significantly associated with increased risk of cervical cancer in Taiwanese women [5]. ITPR3 has been shown to participate in induction of apoptosis in T lymphocytes and other types of cells [6-8]. The expression level of ITPR 3 is associated with growth and aggressiveness of different types of tumors [9-12]. In addition, a number of studies have demonstrated that specific single nucleotide polymorphisms (SNPs) in the ITPR 3 gene were associated with susceptibility to various immune-mediated diseases, such as type 1 diabetes, systemic lupus erythematosus, rheumatoid arthritis, and Graves' disease [13-15].

Because of the importance of ITPR 3 to the immunity and development of immune-associated diseases, it is reasonable to speculate that ITPR 3 genetic variants might participate in the cancer pathogenesis. We therefore tested the hypothesis that specific ITPR3 SNPs are associated with cervical cancer risk with an association study of 462 cervical squamous cell carcinoma (CSCC) patients and 921 healthy controls.

\section{RESULTS}

A total of $84.7 \%$ detection rate for HPV DNA was found in 462 CSCC samples. The HPV type distributions were observed that HPV 16 was $63.5 \%$, HPV 18 was $9.8 \%$, and the remaining types were $26.7 \%$.

The genotype and allele distributions of ITPR $3 \mathrm{SNPs}$ in cases and controls were shown in Tables 1 and 2. The genotype frequencies of all SNPs in the control group did not deviate significantly from Hardy-Weinberg equilibrium $(P>0.05)$. We did not find any significant differences in the genotype and allele frequencies for the 2 ITPR 3 SNPs between CSCC patients and controls (Tables 1 and 2).
Based on the HPV-16 positivity stratification analysis, the synergistic effect of HPV-16 infection and P2RX7 polymorphisms on the risk of CSCC can be explored. The results showed that no significant difference of genotype and allele distributions was observed between women with HPV-16 positive CSCC and controls (Tables 1 and 2). Linkage disequilibrium (LD) analyses between the 2 SNPs revealed strong LDs in both controls $\left(D^{\prime}=0.87\right)$ and patients $\left(D^{\prime}=0.72\right)$.

The inferred haplotypes constructed by $r s 3748079$ $A / G$ and $r s 2229634 C / T$ SNPs were shown in Table 3. The overall $P$ values achieved statistical significances $\left(P=7.0 \times 10^{-3}\right.$ and $4.8 \times 10^{-3}$ in the CSCC and HPV-16 positive CSCC groups, respectively). We found $G T$ was the most frequent but was not associated with CSCC after Bonferroni corrections. However, the frequencies of $A T$ haplotype increased significantly in both CSCC patients $\left(\mathrm{OR}=2.28,95 \% \mathrm{CI} 1.31-3.97, P=2.83 \times 10^{-3}\right)$ and HPV16 positive $\mathrm{CSCC}$ patients $(\mathrm{OR}=2.89,95 \%$ CI $1.55-5.37$, $\left.P=4.54 \times 10^{-4}\right)$, which remained significant even after correction for multiple comparisons $\left(P_{c}=0.01\right.$ and $1.81 \times$ $10^{-3}$, respectively) (Table 3 ).

\section{DISCUSSION}

In this study, we investigated the association of ITPR $3 r s 3748079 \mathrm{~A} / G$ and $r s 2229634 \mathrm{C} / T$ SNPs and their haplotypes with the cervical cancer risk in Taiwanese women. The rationale for choosing these 2 variants is based on their potential functional implication in ITPR 3 gene. The rs 3748079 SNP, located in promoter region of ITPR 3 gene, has been found to upregulate ITPR 3 mRNA expression in cells having $A$ allele [14]. Another variant, $r s 2229634$, is a synonymous SNP situated in exon 20, 
Table 2: Genotype and allele frequencies of the ITPR3 rs2229634 C/T polymorphism in controls and in women with CSCC and those with HPV-16 positive CSCC*

\begin{tabular}{cccccccc}
\hline & $\begin{array}{c}\text { Controls } \\
(\mathbf{N}=\mathbf{9 2 1})\end{array}$ & $\begin{array}{c}\text { CSCC } \\
(\mathbf{N}=\mathbf{4 6 2})\end{array}$ & $\begin{array}{c}\text { HPV-16 } \\
\text { positive } \\
\text { CSCC } \\
(\mathbf{N}=\mathbf{2 4 5})\end{array}$ & & CSCC & HPV-16 positive CSCC \\
\hline & No. (\%) & No. $(\%)$ & No. $(\%)$ & $\boldsymbol{P}$ value $\left(\chi^{2}\right)$ & OR (95\% CI) & P value $\left(\chi^{2}\right)$ & OR (95\% CI) \\
\hline Genotype & & & & $0.23(2.90)$ & & $0.19(3.34)$ & \\
C/C & $251(27.3)$ & $146(31.6)$ & $75(30.6)$ & & $1.23(0.97-1.57)$ & & $1.18(0.87-1.60)$ \\
C/T & $440(47.8)$ & $205(44.4)$ & $101(41.2)$ & & $0.87(0.70-1.09)$ & & $0.77(0.58-1.02)$ \\
T/T & $230(24.9)$ & $111(24.0)$ & $69(28.2)$ & & $0.95(0.73-1.23)$ & & $1.18(0.86-1.62)$ \\
Allele & & & & $0.19(1.73)$ & & $0.97(0.00)$ & \\
C & $942(51.1)$ & $497(53.8)$ & $251(51.2)$ & & $1.11(0.95-1.30)$ & & $1.00(0.82-1.22)$ \\
T & $900(48.9)$ & $427(46.2)$ & $239(48.8)$ & & $0.90(0.77-1.05)$ & & $1.00(0.82-1.22)$ \\
\hline
\end{tabular}

* ITPR3 = inositol 1,4,5-trisphosphate receptor type 3; CSCC = cervical squamous cell carcinoma; HPV = human papillomavirus; $\mathrm{OR}=$ odds ratio; $\mathrm{CI}=$ confidence interval.

which might affect mRNA stability and splicing [16]. We found that, individually, rs3748079 and rs2229634 SNPs were not associated with CSCC susceptibility. However, the haplotype rs3748079 A-rs2229634 T conferred a risk of CSCC patients and the risk increased further in CSCC patients infected with HPV-16. The limitations of our study include a selection bias existing in the study of retrospective design and scarcity of screened SNPs. Therefore, a large-scale prospective study that investigates other SNPs of ITPR3 genes is needed to confirm our findings.

$\mathrm{Ca}^{2+}$ serves as a universal second messenger in virtually all cells. It plays a central role in regulating proliferation/activation and apoptosis of both immune and cancer cells $[17,18]$. When cells are challenged with diverse extracellular stimuli, phospholipase $\mathrm{C}$ pathway is activated and results in generation of IP3, which binds to IP3 receptors and releases $\mathrm{Ca}^{2+}$ from the endoplasmic reticulum. IP3 receptors are a principal route of $\mathrm{Ca}^{2+}$ flux and this makes them an excellent candidate for immune- and cancer-associated studies. An investigation performed by Khan et al. found that increased ITPR3 may be causally related to lymphocyte apoptosis [6]. Other reports showed that ITPR1 and ITPR3 are critical for T-cell activation because of their involvement in IL-2 and IFN- $\gamma$ production $[19,20]$. In addition, a few studies found that overexpression of ITPR 3 in breast and colon cancer cells decreased apoptosis, while knockdown of the receptor enhanced apoptosis $[9,10]$. Cancer cells also exploit various kinds of proto-oncogenes and tumorsuppressors to modulate the expression and function of IP3 receptors in their favor [21]. These findings strongly support a potential role for ITPR3 in carcinogenesis. However, the associations between individual ITPR 3 gene polymorphism and CSCC risk have not been discovered in our study.

A haplotype is a set of DNA variations along a chromosome, which tends to be inherited together. A few studies have found that haplotypes may contribute to a phenotype through the combined effects of multiple individual SNPs on promoter activity or protein function $[22,23]$. The hypothesis that haplotypes are more important than SNPs has been supported by both simulated and empirical data. A simulation study reported by Akey et al. showed that haplotypes can significantly improve the power of an association test [24]. They also used published data from the hereditary hemochromatosis disease region to validate their findings. Another study conducted by Zhang et al. used coalescent simulations to infer haplotype frequencies and quantitatively assess the power in both case-control and case-parental control designs [25]. They found that haplotype-based analysis is always more powerful than marker-by-marker analysis. Fallin et al. applied expectation-maximization algorithm estimated haplotype frequencies to investigate associations between apolipoprotein E SNPs and Alzheimer's disease [26]. They showed that significant associations were obtained for haplotypes that were not identified using individual SNPs. These results clearly provide examples that haplotype analysis is more informative than association studies based on a single marker.

To the best of our knowledge, this study is the first to explore the association between ITPR 3 gene haplotypes and cervical cancer. The $A T$ haplotype was found to be associated with risk of women with CSCC and HPV-16 positive CSCC even though the $r s 3748079 \mathrm{~A} / \mathrm{G}$ or $r s 2229634 \mathrm{C} / \mathrm{T}$ polymorphism alone was not. Thus, we speculate that the haplotype $A T$ may 
Table 3: Analysis of ITPR3 haplotypes in controls and in women with CSCC and those with HPV-16 positive CSCC*

$\begin{array}{ccccc}\text { Controls } & \text { CSCC } & \text { HPV-16 } & \text { CSCC } & \text { HPV-16 positive CSCC } \\ (2 \mathrm{~N}=1842) & (2 \mathrm{~N}=924) & \text { positive } & \\ & & \text { CSCC } & \\ & & (2 \mathrm{~N}=490) & \end{array}$

$(2 \mathrm{~N}=490)$

\begin{tabular}{lccccccc}
\hline Haplotype & No. $(\%)$ & No. $(\%)$ & No. $(\%)$ & $\boldsymbol{P}$ value $\left(\chi^{2}\right)$ & OR $(\mathbf{9 5 \%}$ CI) & $P$ value $\left(\chi^{2}\right)$ & OR $(\mathbf{9 5 \%}$ CI) \\
\hline GT & $875(47.5)$ & $400(43.3)$ & $221(45.0)$ & $0.04(4.39)$ & $0.84(0.72-0.99)$ & $0.34(0.90)$ & $0.91(0.74-1.11)$ \\
GC & $586(31.8)$ & $314(34.0)$ & $161(32.9)$ & $0.25(1.32)$ & $1.10(0.93-1.30)$ & $0.66(0.19)$ & $1.05(0.85-1.30)$ \\
AC & $357(19.4)$ & $183(19.8)$ & $90(18.3)$ & $0.79(0.07)$ & $1.03(0.84-1.25)$ & $0.61(0.26)$ & $0.94(0.72-1.21)$ \\
AT & $24(1.3)$ & $27(2.9)$ & $18(3.7)$ & $\begin{array}{c}2.83 \times 10^{-3} \\
(8.91)\end{array}$ & $2.28(1.31-3.97)$ & $\begin{array}{c}4.54 \times 10^{-4} \\
(12.3)\end{array}$ & $2.89(1.55-5.37)$ \\
\hline
\end{tabular}

* Haplotype inferred using Haploview 4.2 program, based on the order of $r s 3748079$ A/G and $r s 2229634 C / T$ polymorphisms.

ITPR3 = inositol 1,4,5-trisphosphate receptor type 3; CSCC = cervical squamous cell carcinoma; HPV = human papillomavirus; $\mathrm{OR}=$ odds ratio; $\mathrm{CI}=$ confidence interval.

$P$ value for 4 haplotypes between all CSCC patients and controls: $P=7.0 \times 10^{-3}\left(\chi^{2}=12.1,3 \mathrm{df}\right)$.

$P$ value for 4 haplotypes between HPV-16 positive CSCC patients and controls: $P=4.8 \times 10^{-3}\left(\chi^{2}=12.9,3 \mathrm{df}\right)$.

have a role in ITPR 3 gene regulation and function and involve in a mechanism that determines the outcome of HPV infection and the subsequent development of CSCC. There are several possible explanations for these findings. The interaction of multiple SNPs within a haplotype may ultimately produce the biologic phenotype and become a powerful determinant of disease susceptibility. Another possibility is that the specific $A T$ haplotype found in this study is in LD with the class II HLA loci.

Because ITPR 3 locates at about $500 \mathrm{~kb}$ centrometric to the class II $H L A$ genes DR and DQ, which are found to associate with cervical cancer $[27,28]$, it is important to clarify whether association between ITPR 3 AT haplotype and CSCC is dependent on LD with class II HLA genes. The LD between ITPR 3 and HLA-DRB1 alleles was analyzed in the subgroup of 333 CSCC patients and 241 controls using the PyPop software [29]. In this subgroup, we did not find any significant associations between $H L A-D R B 1$ alleles and CSCC. Only weak LD was identified between ITPR 3 and $H L A-D R B 1$ alleles in the control subset $\left(D^{\prime}=0.31\right.$ and 0.34 , respectively). These lines of evidence suggest that it is unlikely that the ITPR 3 AT haplotype association with CSCC is dependent on $H L A-D R B 1$ locus. However, we are unable to exclude the LD effect of $H L A-D Q B 1$ alleles or DRB1-DQB1 haplotypes on the association of ITPR $3 A T$ haplotype with CSCC.

In summary, our study demonstrates that the $A T$ haplotype in ITPR3 gene is markedly associated with CSCC risk in Taiwanese women. Additionally, women carrying the $A T$ haplotype and harboring HPV-16 infection enhance susceptibility to CSCC. Further investigations are warranted to elucidate the specific role of ITPR3 molecule on the etiology of CSCC.

\section{PATIENTS AND METHODS}

\section{Study subjects}

The study included 462 patients with CSCC (mean \pm SD age at diagnosis: $53.2 \pm 13.1$ years) residing in northern Taiwan. The diagnosis of CSCC was confirmed by histological examinations of tissues from biopsies or resected specimens. The control group consisted of 921 sex- and age-matched healthy subjects (mean \pm SD age at sampling: $52.4 \pm 12.1$ years) who were enrolled from women attending the routine Pap screening and with normal Pap smear and no previous history of cervical dysplasia. The cases and controls were all genetically unrelated Taiwanese women. Informed written consent was obtained from all participants for use of their surgical resections or cervical scrapings for the study. The study protocol conformed to the ethical guidelines of the 1964 Declaration of Helsinki and was approved by the Institutional Review Board of Mackay Memorial Hospital.

\section{DNA extraction}

Formalin-fixed, paraffin-embedded tissue blocks from CSCC patients were sectioned and dewaxed, and genomic DNA was then extracted using the Qiagen DNeasy Tissue Kit (Qiagen, Valencia, CA) according to the manufacturer's protocol. Genomic DNA of controls was extracted from cervical scrapings using Qiagen DNA extraction kit.

\section{HPV detection and typing}

The detection of HPV and genotyping on 462 cervical DNA samples were performed by polymerase 
chain reaction (PCR). A pair of degenerate primers, GP6+/MY11, designed according to the highly conserved domain, was used to amplify a fragment of approximately $192 \mathrm{bp}$ in the L1 region of the HPV genome [30, 31]. The PCR product was then sequenced on an automated sequencer (ABI 377, Applied Biosystems, Foster City, CA) to determine the HPV genotype. Since stratifications based on HPV types in controls were not performed in this study, no HPV DNA testing was done for the 921 control subjects.

\section{ITPR3 genotyping}

ITPR3 rs3748079 $A / G$ and $r s 2229634 C / T$ polymorphisms were genotyped. They were determined using the Pre-Developed TaqMan Allelic Discrimination Assay (Applied Biosystems, Foster City, CA). Briefly, polymerase chain reactions (PCR) were carried out in a 96well GeneAmp PCR System 9700 (Applied Biosystems) with mixes consisting of $10 \mathrm{ng}$ of genomic DNA, $5 \mu \mathrm{l}$ of TaqMan Universal PCR Master Mix, $0.5 \mu$ of $20 \times$ Assay Mix, and $\mathrm{ddH}_{2} \mathrm{O}$ to a final volume of $10 \mu \mathrm{l}$. Thermal cycle conditions were as follows: denaturation at $95^{\circ} \mathrm{C}$ for 10 min, followed by 40 cycles of denaturation at $92^{\circ} \mathrm{C}$ for 15 sec, and annealing and extension at $60^{\circ} \mathrm{C}$ for $1 \mathrm{~min}$. After PCR, the TaqMan assay plates were transferred to the ABI PRISM 7000 Sequence Detection System (Applied Biosystems) where the endpoint fluorescence intensity in each well of the plate was read. The allele specific fluorescence data from each plate were analyzed using the SDS v.1.1 software (Applied Biosystems) to automatically determine the genotype of each sample.

\section{Statistical analysis}

Genotype and allele frequencies of the ITPR3 $r s 3748079 \mathrm{~A} / \mathrm{G}$ and $r s 2229634 \mathrm{C} / \mathrm{T}$ SNPs were determined by direct counting. The Hardy-Weinberg equilibrium was assessed for each SNP in both the control and case groups by chi-square analysis. Linkage analysis and the frequencies of ITPR 3 haplotypes in controls and cases were estimated using the Haploview 4.2 program [32].

Statistical differences in the genotype, allele, and haplotype distributions between controls and cases were performed by chi-square test with Yates' correction or Fisher's exact test (when the number of subjects in a cell was $<5$ ). Odds ratios (OR) and 95\% confidence intervals (CI) were also calculated. Bonferroni correction for multiple testing was applied by multiplying $P$ value with the number of comparisons performed. $P_{c}$ values of less than 0.05 (2-tailed) were considered to be statistically significant.

Prior to the study, statistical power to detect effects of the ITPR3 SNPs on susceptibility to CSCC was calculated using the Quanto Ver. 1.1 software (Department of Preventive Medicine, University of Southern California, CA, USA). We designed the study to have a power $>95 \%$ to determine a relative risk of 1.4 for the genotype of each SNP at a significance level of 0.05 , with an estimated prevalence of CSCC of 360/100,000 [33].

\section{ACKNOWLEDGMENTS}

This research was supported by grants MMH 9781 and MMH E-105-07 from Mackay Memorial Hospital, Taiwan.

\section{CONFLICTS OF INTEREST}

The authors declare that they have no conflict of interest.

\section{REFERENCES}

1. Chen CA, and Hsieh CY. Recent advances and problems in primary therapy for cervical cancer in Taiwan. J Formos Med Assoc. 2004; 103: 511-8.

2. Munoz N. Human papillomavirus and cancer: the epidemiological evidence. J Clin Virol. 2000; 19: 1-5.

3. Patterson RL, Boehning D, and Snyder SH. Inositol 1,4,5-trisphosphate receptors as signal integrators. Annu Rev Biochem. 2004; 73: 437-65.

4. Imboden JB, and Pattison G. Regulation of inositol 1,4,5-trisphosphate kinase activity after stimulation of human T cell antigen receptor. J Clin Invest. 1987; 79: 1538-41.

5. Yang YC, Chang TY, Chen TC, Chang SC, Chen WF, Chan HW, Lin WS, Wu FT, and Lee YJ. Genetic polymorphisms in the ITPKC gene and cervical squamous cell carcinoma risk. Cancer Immunol Immunother. 2012; 61: 2153-9.

6. Khan AA, Soloski MJ, Sharp AH, Schilling G, Sabatini DM, Li SH, Ross CA, and Snyder SH. Lymphocyte apoptosis: mediation by increased type 3 inositol 1,4,5-trisphosphate receptor. Science. 1996; 273: 503-7.

7. Blackshaw S, Sawa A, Sharp AH, Ross CA, Snyder SH, and Khan AA. Type 3 inositol 1,4,5-trisphosphate receptor modulates cell death. Faseb J. 2000; 14: 1375-9.

8. Mendes CC, Gomes DA, Thompson M, Souto NC, Goes TS, Goes AM, Rodrigues MA, Gomez MV, Nathanson $\mathrm{MH}$, and Leite MF. The type III inositol 1,4,5-trisphosphate receptor preferentially transmits apoptotic $\mathrm{Ca} 2+$ signals into mitochondria. J Biol Chem. 2005; 280: 40892-900.

9. Szatkowski C, Parys JB, Ouadid-Ahidouch H, and Matifat F. Inositol 1,4,5-trisphosphate-induced $\mathrm{Ca} 2+$ signalling is involved in estradiol-induced breast cancer epithelial cell growth. Mol Cancer. 2010; 9: 156.

10. Shibao K, Fiedler MJ, Nagata J, Minagawa N, Hirata K, Nakayama Y, Iwakiri Y, Nathanson MH, and Yamaguchi $\mathrm{K}$. The type III inositol 1,4,5-trisphosphate receptor is associated with aggressiveness of colorectal carcinoma. Cell Calcium. 2010; 48: 315-23. 
11. Kang SS, Han KS, Ku BM, et al. Caffeine-mediated inhibition of calcium release channel inositol 1,4,5-trisphosphate receptor subtype 3 blocks glioblastoma invasion and extends survival. Cancer Res. 2010; 70: 1173-83.

12. Sakakura C, Hagiwara A, Fukuda K, Shimomura K, Takagi T, Kin S, Nakase Y, Fujiyama J, Mikoshiba K, Okazaki $\mathrm{Y}$, and Yamagishi H. Possible involvement of inositol 1,4,5-trisphosphate receptor type 3 (IP3R3) in the peritoneal dissemination of gastric cancers. Anticancer Res. 2003; 23: 3691-7.

13. Roach JC, Deutsch K, Li S, Siegel AF, Bekris LM, Einhaus DC, Sheridan CM, Glusman G, Hood L, Lernmark A, and Janer M. Genetic mapping at 3-kilobase resolution reveals inositol 1,4,5-triphosphate receptor 3 as a risk factor for type 1 diabetes in Sweden. Am J Hum Genet. 2006; 79: 614-27.

14. Oishi T, Iida A, Otsubo S, et al. A functional SNP in the NKX2.5-binding site of ITPR3 promoter is associated with susceptibility to systemic lupus erythematosus in Japanese population. J Hum Genet. 2008; 53: 151-62.

15. Nakabayashi K, Tajima A, Yamamoto K, et al. Identification of independent risk loci for Graves' disease within the MHC in the Japanese population. J Hum Genet. 2011; 56: 772-8.

16. Chamary JV, Parmley JL, and Hurst LD. Hearing silence: non-neutral evolution at synonymous sites in mammals. Nat Rev Genet. 2006; 7: 98-108.

17. Feske S. Calcium signalling in lymphocyte activation and disease. Nat Rev Immunol. 2007; 7: 690-702.

18. Capiod T, Shuba Y, Skryma R, and Prevarskaya N. Calcium signalling and cancer cell growth. Subcell Biochem. 2007; 45: 405-27.

19. Jayaraman T, Ondriasova E, Ondrias K, Harnick DJ, and Marks AR. The inositol 1,4,5-trisphosphate receptor is essential for T-cell receptor signaling. Proc Natl Acad Sci U S A. 1995; 92: 6007-11.

20. Nagaleekar VK, Diehl SA, Juncadella I, Charland C, Muthusamy N, Eaton S, Haynes L, Garrett-Sinha LA, Anguita J, and Rincon M. IP3 receptor-mediated Ca2+ release in naive $\mathrm{CD} 4 \mathrm{~T}$ cells dictates their cytokine program. J Immunol. 2008; 181: 8315-22.

21. Akl H, and Bultynck G. Altered $\mathrm{Ca}(2+)$ signaling in cancer cells: proto-oncogenes and tumor suppressors targeting IP3 receptors. Biochim Biophys Acta. 2013; 1835: 180-93.

22. Drysdale CM, McGraw DW, Stack CB, Stephens JC, Judson RS, Nandabalan K, Arnold K, Ruano G, and Liggett $\mathrm{SB}$. Complex promoter and coding region beta 2-adrenergic receptor haplotypes alter receptor expression and predict in vivo responsiveness. Proc Natl Acad Sci U S A. 2000; 97: 10483-8.

23. Joosten PH, Toepoel M, Mariman EC, and Van Zoelen EJ. Promoter haplotype combinations of the platelet-derived growth factor alpha-receptor gene predispose to human neural tube defects. Nat Genet. 2001; 27: 215-7.

24. Akey J, Jin L, and Xiong M. Haplotypes vs single marker linkage disequilibrium tests: what do we gain? Eur J Hum Genet. 2001; 9: 291-300.

25. Zhang K, Calabrese P, Nordborg M, and Sun F. Haplotype block structure and its applications to association studies: power and study designs. Am J Hum Genet. 2002; 71: 1386-94.

26. Fallin D, Cohen A, Essioux L, Chumakov I, Blumenfeld M, Cohen D, and Schork NJ. Genetic analysis of case/control data using estimated haplotype frequencies: application to APOE locus variation and Alzheimer's disease. Genome Res. 2001; 11: 143-51.

27. Wei LZ, Wang HL, Liu X, Lu YP, Xu F, Yuan JQ, and Ling YQ. Meta-analysis on the relationship between HLADRB1 gene polymorphism and cervical cancer in Chinese population. PLoS ONE. 2014; 9: e88439.

28. Zhang X, Lv Z, Yu H, Wang F, and Zhu J. The HLA-DQB1 gene polymorphisms associated with cervical cancer risk: A meta-analysis. Biomedicine \& Pharmacotherapy. 2015; 73: 58-64.

29. Lancaster AK, Single RM, Solberg OD, Nelson MP, and Thomson G. PyPop update--a software pipeline for largescale multilocus population genomics. Tissue Antigens. 2007; 69: 192-7.

30. Hildesheim A, Schiffman MH, Gravitt PE, Glass AG, Greer CE, Zhang T, Scott DR, Rush BB, Lawler P, Sherman ME, Kurman RJ, and Manos MM. Persistence of type-specific human papillomavirus infection among cytologically normal women. J Infect Dis. 1994; 169: 235-40.

31. de Roda Husman AM, Walboomers JM, van den Brule AJ, Meijer CJ, and Snijders PJ. The use of general primers GP5 and GP6 elongated at their 3' ends with adjacent highly conserved sequences improves human papillomavirus detection by PCR. J Gen Virol. 1995; 76: 1057-62.

32. Barrett JC, Fry B, Maller J, and Daly MJ. Haploview: analysis and visualization of $\mathrm{LD}$ and haplotype maps. Bioinformatics. 2005; 21: 263-5.

33. Chen YY, You SL, Chen CA, Shih LY, Koong SL, Chao KY, Hsiao ML, Hsieh CY, and Chen CJ. Effectiveness of national cervical cancer screening programme in Taiwan: 12-year experiences. Br J Cancer. 2009; 101: 174-7. 